\title{
GESTIÓN Y PRÁCTICAS DE LA RESPONSABILIDAD SOCIAL CORPORATIVA EN AMÉRICA LATINA DURANTE LA PANDEMIA DEL COVID-19*
}

\section{Management and practices of corporate social responsibility in Latin America during the COVID-19 pandemic}

Martha Rocío Godoy Rodríguez**, Aiblis Susel Vidal Marrero***, Juan Pablo Cendales Rodríguez ${ }^{* * * *}$, Carolina Asuaga*****, José Obdulio Curvelo Hassán ${ }^{* * * * *}$

Recepción: 03 de marzo de 2021. Aceptación: 7 de julio de 2021

DOI: http://dx.doi.org/10.21017/Rev.Repub.2021.v31.a115

* Artículo producto de investigación resultado de las estrategias de colaboración y cohesión entre los Grupos de Investigación GAFEC, contabilidad y entorno social y el grupo de investigación de la Facultad de ciencias económicas y de administración? de la Universidad de la República del Uruguay. Su contribución se centra en la compresión de los fenómenos sociales que afectaron a las empresas en América Latina a partir de la pandemia generada por el COVID-19, sobre los cuales existe una literatura muy limitada. La financiación de la investigación estuvo a cargo del Comité Nacional para el Desarrollo de la Investigación -CONADI- de la Universidad Cooperativa de Colombia, la Universidad de la Republica del Uruguay y el Fondo de investigación de la Corporación Universitaria Republicana.

** Docente de tiempo completo de la Facultad de Contaduría Pública de la Universidad Cooperativa de Colombia en Bogotá. Magíster en Pensamiento estratégico y prospectivo, de la Universidad Externado de Colombia. Especialista en Alta Gerencia por la Universidad Cooperativa de Colombia. Profesional en Administración Financiera de la Universidad del Tolima. ORCID: https:/ / orcid.org0000-0002-3194-806X; correo electrónico: martha.godoy@campusucc.edu.co

*** Docente en la Universidad de la República (Montevideo, Uruguay). Máster en Administración de Negocios de la Universidad de Camagüey, Cuba. Licenciada en Contabilidad y Finanzas de la Universidad de Las Tunas, Cuba. ORCID: https:/ / orcid.org/ 0000-0001-5882-3673, correo electrónico: avidal@ccee.edu.uy

**** Docente de tiempo completo de la Facultad de Contaduría Pública de la Universidad Cooperativa de Colombia en Bogotá. Magíster en Administración de empresas y contador público de la Universidad Nacional de Colombia en Bogotá. ORCID: https:/ /orcid.org/0000-0003-2384-0224; correo electrónico: juan.cendalesr@campusucc.edu.co

***** Profesora titular de la Universidad de la República (Montevideo, Uruguay) en Costos y Control de Gestión y coordinadora académica del Posgrado en Costos y Gestión Empresarial. Máster (DEA) en Economía Financiera y Contabilidad de la Universidad de Castilla-La Mancha. Especialización en Turismo para el Mercosur de Japan International Cooperation Agency (JICA). Especialista en Costos y Gestión Empresarial de la Universidad Nacional de La Plata. Contadora Pública de la Universidad de la República de Uruguay. ORCID: https:/ / orcid.org/0000-0002-4454-4152; correo electrónico: casuaga@ccee.edu.uy

****** Investigador del grupo GACEF (Bogotá, Colombia). Doctor en Contabilidad por la Universidad de Valencia. Magíster en filosofía por la Universidad Santo Tomás. Especialista en Revisoría Fiscal y contador público por la Universidad Cooperativa de Colombia. ORCID: https:// orcid.org/0000-0002-6397-9450; correo electrónico: : jcurvelo@urepublicana.edu.co 


\section{RESUMEN}

El escenario del COVID-19 condujo a nuevas formas de comprar, vender y consumir, triada que hizo emerger apuestas voluntarias por parte de las empresas para el mejoramiento social, económico y ambiental que legitimara su accionar ante el nuevo contexto. Este proceso legitimario en situaciones como el confinamiento, el trabajo en casa, la interacción con equipos diversos e, incluso, la transformación de las prácticas productivas ante la escasez de materias primas condujo a una perspectiva conductual de la responsabilidad social corporativa (RSC) centrada en la capacidad adaptativa a partir del entender las dinámicas del contexto.

Bajo este entender, la presente investigación caracteriza la gestión y las prácticas de la RSC durante la pandemia en América Latina. Metodológicamente se estudiaron las 200 empresas mejor posicionadas en el Ranking Merco, que permite una interacción de empresas, líderes, y responsabilidad y gobierno corporativo. Se estudió la variable «aporte social» con 8 variables cruzadas y se contrastó con la información divulgada por las organizaciones estudiadas respecto a su gestión y prácticas de RSC en el periodo de la pandemia. Los países seleccionados fueron Uruguay y Colombia, atendiendo su ubicación geográfica extrema en el continente, la disparidad en el PIB y la diferencia en las políticas estatales para gestionar la pandemia.

Tras inferir las hipótesis de la investigación se encuentra que las empresas estudiadas, pese a la disparidad territorial, aspectos geográficos, número habitantes y gestión por separado de la pandemia, tanto en Colombia como en Uruguay, adoptaron medidas importantes para enfrentar la crisis producida por el COVID-19. Se destaca el aporte social del 92\% de las empresas de Uruguay y el 99\% de Colombia a nuevos modelos de negocio, a la digitalización de operaciones, entrega de canastas de alimentos e higiene personal, aportes tecnológicos y entre otros, donaciones de insumos y equipos médicos. Las empresas que más aportaron están ubicadas en los primeros puestos del ranking de sus países y pertenecen al sector industrial en su mayoría, sector que en principio presenta mayor afectación por la pandemia. Se aúna a lo anterior que las empresas más longevas realizaron mayores contribuciones. La totalidad de las estudiadas presentó informes de sostenibilidad empleando estándares e indicadores internacionales y focalizaron las aportaciones sociales y ambientales desde inicio de la pandemia.

Palabras clave: COVID-19, responsabilidad social corporativa, ética, empresa.

\section{ABSTRACT}

The COVID-19 scenario led to new ways of buying, selling, and consuming, a triad that lead to emerging voluntary bets on the part of companies for social, economic, and environmental improvement that legitimizes their actions in the 
new context. This mentioned process in situations such as confinement, work at home, interaction with diverse teams, and even the transformation of productive practices in the face of the scarcity of raw materials led to a behavioral perspective of corporate social responsibility (CSR) focused on the adaptative capacity of the dynamics of the context.

Under this understanding, this research characterizes the management and practices of CSR during the pandemic in Latin America. Methodologically, the 200 best-positioned companies in the Merco Ranking were studied, allowing interaction of companies, leaders, and corporate responsibility and governance. The «social contribution» variable was analyzed with eight crossed variables and contrasted with the information disclosed by the organizations have studied regarding management and CSR practices in the period of the pandemic. The selected countries were Uruguay and Colombia, considering their extreme geographic location on the continent, the disparity in GDP, and the difference in state policies to manage the pandemic.

After inferring the research hypothesis, it was possible to determine that the companies studied, despite the territorial disparity, geographical aspects, the number of inhabitants, and separate management of the pandemic, both in Colombia and Uruguay, adopted several measures to face the crisis produced by the COVID-19. Additionally, a social contribution has in both markets has been made, where $92 \%$ of Uruguayan companies and $99 \%$ of Colombia companies have developed new business models. The mentioned models include digitization of operations, delivery of food baskets and personal hygiene, technological contributions, and among others, donations of supplies and medical pieces of equipment.

The companies that contributed the most are located at the top of the ranking in their countries and mostly belong to the industrial sector, a sector that in principle is most affected by the pandemic. In addition, the longest-standing companies made greater contributions. All of those studied presented sustainability reports using international standards and indicators and focused on social and environmental contributions since the beginning of the pandemic.

Key words: COVID-19, corporate social responsibility, ethics, business.

\section{INTRODUCCIÓN}

En los últimos años se ha instaurado el debate sobre qué entender por RSC y en qué contexto entenderlo. Si bien esta discusión deviene de una tradición de la ética aplicada, sus condicionantes siguen estando reservados a un campo propio de la moral, como lo evidencia Fischer (2004). A ello se debe agregar que con el giro lingüístico pragmático (Wittgenstein, 2014) se empieza a considerar que quienes poseen actos de habla tienen obligaciones éticas; siendo así, las empresas deben asumir prácticas responsables dando cuenta en qué contexto y en qué tipo de sociedad las asumen. 
Estos consensos conducen a considerar la RSC como una manifestación pública sobre las prácticas corporativas, que las mismas son realizadas de forma transparente, que los recursos utilizados en su desarrollo misional consideran su agotamiento y entre otras, que actúan conforme a un sistema de valores reconocidos por la sociedad. Ello conduce a que las organizaciones asuman una actitud reflexiva y crítica con el entorno, para generar respuestas asertivas y oportunidad a las manifestaciones y las dinámicas asociadas a la sostenibilidad.

En este orden de ideas es factible afirmar que las organizaciones deben generar un entorno moralmente solidario que reconozca las aspiraciones y compromisos de una determinada sociedad. Frente al escenario actual de la pandemia generada por el SARS-COV-2 (COVID-19) se espera que la gestión y las prácticas de la RSC den cuenta de un nuevo contexto generado con la digitalización, los cambios en la producción y el nuevo relacionamiento humano (Rendtorff, 2009). Se trata de una motivación ética alrededor de la conservación de las formas de vida humana y no humana (Jonas, 2014), de la preservación de las especies, de los nuevos consumos y de la manera como el aparato productivo media entre avanzar en sus operaciones rutinarias y contribuir a garantizar las condiciones vitales.

He y Harris (2020) afirman que la lectura del contexto generado por el SARSCOV-2 debe permitir a las organizaciones abordar los desafíos sociales y ambientales globales que han sido, entre otros, acordados en la agenda 2030 y en los Objetivos de Desarrollo Sostenible -ODS- (CEPAL, 2018). Qiu, et al. (2021) hablan de la incidencia de estos fenómenos en el valor de la empresa, aspecto sensible toda vez que la pandemia ha desarrollado reacciones y adaptaciones que se verán reflejadas en nuevos hábitos de los consumidores (Popkova, et al., 2021; Kirk y Rifkin, 2020), en sus nuevas enfermedades psicológicas y biológicas, en sus interacciones sociales y en procesos culturales mediados por el ciberespacio.

En línea con los argumentos desarrollados, asumir lecturas críticas y reflexivas alrededor de la gestión y la práctica de la gestión de la RSC y del quehacer de las empresas en la sociedad a partir de la pandemia del COVID-19 constituye un imperativo. Maritz (2020) encuentra que las empresas que tenían mayor fortaleza en la gestión de la RSC se adaptaron más rápido al cambio, generaron respuestas prontas ante el SARS-COV-2 y promovieron una diversidad de interacciones con el entorno digital. Estas manifestaciones son objeto de grandes transformaciones a partir de la pandemia e implican la interacción con el metaverso, las cripto-divisas, la gestión del conocimiento frente a cambios ambientales extremos y una ampliación de la cadena de suministros centrada en la circulación ciudadana y la nueva interacción social.

Se evidencia así la hibridación de los postulados de la teoría de los stakeholders (Agle, et al., 2008), la teoría de la legitimidad (Archel, 2009), la moderna teoría 
de la firma (Boatright, 1996) y la teoría contractual (Butler, 1998). Estos cambios además de afectar la memoria de sostenibilidad también lo hacen en la información financiera, siguiendo las consideraciones de la teoría de la agencia (Watts y Zimmerman, 1978), y apropiados en los términos dispuestos en las regulaciones sobre revelaciones y las contribuciones a los ODS - objetivo 12 en su meta $6-$. La apreciación de estas teorías lleva a formular las siguientes hipótesis de investigación:

H1: Las organizaciones que asumieron la RSC como un canal de comprensión de las dinámicas del entorno para generar respuestas adaptativas son aquellas que han respondido de mejor manera ante la pandemia generada por el COVID-19.

H2: Las prácticas de la RSC en el escenario del COVID-19 se caracterizan por una orientación hacia los Objetivos de Desarrollo Sostenible.

H3: La trayectoria de una organización le permite contar con aprendizajes y capacidades de adaptación a cambios a diferencia de las más jóvenes, que tienen poca capacidad de reacción ante escenarios de incertidumbre o adversos.

\section{METODOLOGÍA}

A fin de contrastar las hipótesis de investigación se seleccionó un grupo de empresas representativas en América Latina en el Ranking Merco, uno de los monitores de referencia en el mundo para la reputación corporativa, tomando como muestra 200 con la mejor posición, 100 de ellas registradas en Uruguay y las 100 restantes en Colombia. Con la entrada del COVID-19 casi de forma simultánea, ambos países mostraron una actuación diferente ante la pandemia y tuvieron cifras de contagios significativamente distintas, lo que motivó a analizar la gestión y las prácticas de la RSC de las empresas seleccionadas bajo el matiz y el contraste de cada coyuntura.

Reconocida a nivel internacional como un caso de éxito en la gestión de la crisis sanitaria, social y económica producida por el coronavirus, la República del Uruguay presenta una de las menores cifras de contagios y muertes por esta enfermedad en la región por cada 100.000 habitantes durante el primer año de la pandemia (datos tomados de Johns Hopkins University $\mathcal{E}$ Medicine, 2020). Estas bajas cifras se lograron gracias a la rápida acción ante la confirmación, el 13 de marzo del 2020, de los primeros casos de coronavirus en el país. Ese mismo día, el gobierno declaró el estado de emergencia sanitaria y adoptó una serie de medidas como el cierre de fronteras y la suspensión 
de vuelos, clases, servicios religiosos y eventos multitudinarios, y se hizo un llamado al aislamiento social. Estas medidas iniciales, sumadas a las acciones posteriores, permitieron que en el país no fuera necesario decretar la cuarentena obligatoria y que, paulatinamente, las actividades suspendidas volvieran a la normalidad.

Cabe destacar que en el ámbito laboral se adoptaron importantes medidas que ayudaron a frenar el avance del virus, como el subsidio especial por desempleo aplicable a todas las actividades, la disminución de la circulación de la población, el subsidio por enfermedad durante el período de cuarentena obligatoria y la inclusión del COVID-19 como enfermedad profesional aplicable al sector salud (PwC Uruguay, 2020). Se destaca la creación del Fondo Coronavirus, financiado principalmente por un recorte del $20 \%$ en los salarios del presidente, los ministros, los legisladores y los empleados públicos superiores a US\$ 1.800 por mes (BBC, 2020).

Por su parte, Colombia muestra en el continente una de las cifras relativas más altas por cada 100.000 habitantes durante el primer año de la pandemia (Johns Hopkins University \& Medicine, 2020), pese a la cuarentena nacional obligatoria decretada desde el 24 de marzo de 2020 y las diversas medidas implantadas, similares a las de Uruguay, como suspensión de clases, prohibiciones de aglomeraciones, cierre de fronteras, restricciones de movilidad y cuarentenas obligatorias y selectivas. Esta situación se debió en parte a su número de habitantes dispersos geográficamente y en condiciones climáticas diferentes, la desigualdad socioeconómica, el alto índice de desempleo, la migración de venezolanos y la crisis política en el país (France 24, 2020).

El gobierno nacional de la República de Colombia generó medidas sociales, económicas, fiscales, comerciales y regulatorias, pero la crisis política que se había generado por el acuerdo de paz en el país y la diversidad ideológica de los recientes gobiernos locales electos (alcaldes de municipio y gobernadores de departamentos) condujo a confrontaciones entre ellos y el gobierno central, complejizando la gestión de la pandemia y agudizando la crisis política que vivía el país, para este caso por las medidas para hacer frente al COVID-19 que, a su juicio, limitaba el protagonismo de ellos como alcaldes y gobernadores.

Justificados los criterios de selección de la muestra, para el análisis del papel de las empresas frente al COVID-19 se parte de confirmar si estas habían hecho contribución para mitigar los efectos causados por la pandemia y si realizaron acciones de RSC en este ámbito. Se acudió a la información divulgada en sus sitios web, artículos de prensa, redes sociales y otros medios de comunicación digital para confirmar dichas contribuciones. Metodológicamente, a par- 
tir de lo anterior, se identificó la variable «aporte social», en torno al cumplimiento del objetivo de la investigación referente a RSC, y luego se analizó la relación existente entre esta y el resto de las variables que se describen en la tabla 1.

Tabla 1. Tipología de variables

\begin{tabular}{|c|c|c|c|}
\hline Nombre & Descripción & & Detalle \\
\hline \multirow{3}{*}{$\begin{array}{l}\text { Aporte } \\
\text { social }\end{array}$} & \multirow{3}{*}{$\begin{array}{c}\text { Aporta para enfrentar el } \\
\text { COVID-19 }\end{array}$} & 0 & No aporta \\
\hline & & 1 & Aporta en menor medida \\
\hline & & 2 & Aporta en mayor medida \\
\hline \multirow{3}{*}{ Ranking } & \multirow{3}{*}{$\begin{array}{l}\text { Mejor reputación } \\
\text { corporativa }\end{array}$} & 1 & Posición 1 \\
\hline & & 2 & Posición 2 \\
\hline & & 3 & Posición $3 \ldots$ \\
\hline \multirow{2}{*}{ Origen } & \multirow{2}{*}{ Nacional/Internacional } & 0 & Nacional \\
\hline & & 1 & Internacional \\
\hline \multirow{7}{*}{ Sector } & \multirow{7}{*}{$\begin{array}{l}\text { Clase de actividad } \\
\text { económica }\end{array}$} & 0 & $\begin{array}{l}\text { Actividades primarias: agricultura, } \\
\text { ganadería, caza, silvicultura, pesca }\end{array}$ \\
\hline & & 1 & Industria \\
\hline & & 2 & Comercio y servicios \\
\hline & & 3 & Construcción \\
\hline & & 4 & Energía, gas y agua \\
\hline & & 5 & Transporte y comunicaciones \\
\hline & & 6 & $\begin{array}{l}\text { Otras actividades (financieras, } \\
\text { inmobiliarias, etc.) }\end{array}$ \\
\hline Antigüedad & Edad de la empresa & & Año de creación \\
\hline Tamaño & Cantidad de trabajadores & & Número de trabajadores \\
\hline \multirow{3}{*}{ Reporte } & \multirow{3}{*}{ Reporta o no reporta } & 0 & No reporta \\
\hline & & 1 & Sí reporta con informe de matriz \\
\hline & & 2 & Sí reporta con informe propio \\
\hline \multirow{5}{*}{$\begin{array}{l}\text { Tipo de } \\
\text { reporte }\end{array}$} & \multirow{5}{*}{$\begin{array}{l}\text { Características del } \\
\text { reporte }\end{array}$} & 1 & Reporte anual \\
\hline & & 2 & Reporte de RS \\
\hline & & 3 & $\begin{array}{l}\text { Reporte con variables sociales, } \\
\text { económicas y ambientales }\end{array}$ \\
\hline & & 4 & $\begin{array}{l}\text { Reporte utilizando estándares } \\
\text { internacionales de RS }\end{array}$ \\
\hline & & 5 & $\begin{array}{l}\text { Reporte utilizando estándares e } \\
\text { indicadores internacionales de RS }\end{array}$ \\
\hline
\end{tabular}

Fuente: Elaboración propia. 


\section{RESULTADOS}

Producto de la correlación de las variables, se encuentra que el $8 \%$ de las empresas estudiadas, radicadas en Uruguay, no declaran ningún aporte para atender la contingencia del COVID-19, según las fuentes consultadas; el 38\% realizan aportes en menor medida que incluyen compartir recomendaciones para prevenir el contagio, adoptan procedimiento internos, crean nuevos modelos de negocio y logran digitalizar sus operaciones; el 54\% restante aportan en mayor medida entregando canastas de alimentos e higiene personal, donaciones de insumos y equipos médicos, y contribuciones monetarias, y producen desarrollos tecnológicos en sus operaciones, como se observa en el gráfico 1.

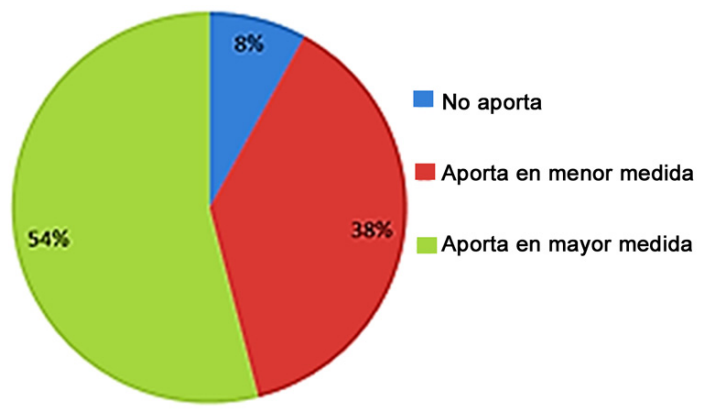

Gráfico 1. Proporción de empresas uruguayas del ranking que contribuyeron a enfrentar la pandemia. Fuente: Elaboración propia

Se adiciona al anterior gráfico que el $38 \%$ presentaron en sus memorias de sostenibilidad información amplia sobre contribuciones para mitigar los efectos de la pandemia. El 54\% adicionaron en su memoria de sostenibilidad los aportes para mediar la pandemia, complementarios a sus estrategias de gestión de la RSC.

Para las empresas radicadas en Colombia la dispersión es menor: todas realizaron contribuciones, destacándose que un 38\% aportó en menor medida; es decir, adoptaron las recomendaciones de la autoridad competente para prevenir el contagio, crearon nuevos modelos de negocio y han logrado digitalizar sus operaciones. El $62 \%$ restante hacen contribución en mayor medida representada en donaciones de insumos y equipos médicos, y contribuciones monetarias, y han producido desarrollos tecnológicos en sus operaciones, como se observa en el gráfico 2. 


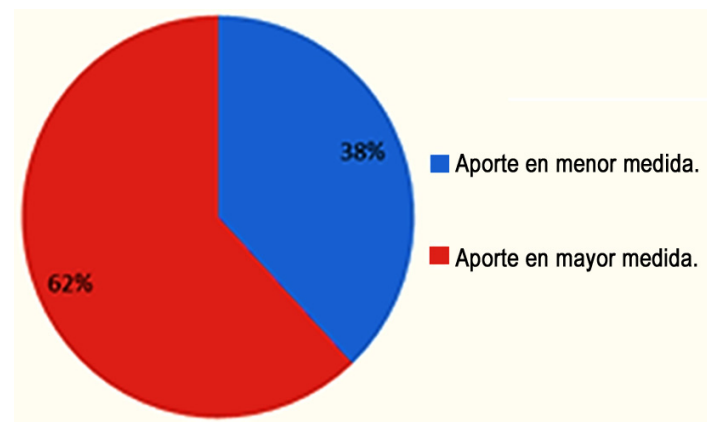

Gráfico 2. Proporción de empresas colombianas del ranking que contribuyeron a enfrentar la pandemia. Fuente: Elaboración propia.

El 38\% de las empresas del anterior gráfico reportan en su memoria de sostenibilidad las estrategias en el marco de la pandemia. El 62\% restante presentaron en sus memorias de sostenibilidad información amplia sobre contribuciones para mitigar los efectos de la pandemia.

No se encontró diferencia en las empresas que divulgan la memoria de manera consolidada en la matriz y entre aquellas que hacen sus informes propios. La relación existente entre el aporte de las empresas y cada una de las variables definidas arroja como resultado que tanto en Uruguay como en Colombia las empresas que más aportaron se ubican en la posición media de raking MERCO como se muestra en los gráficos 3 y 4.

En los anteriores gráficos se puede leer que si bien no es condicionante que una empresa disponga de una alta ubicación en el ranking - posición 1 a la 30 - para llevar a cabo acciones RSC en la pandemia, sí se aprecia que las que se encuentran menos rankeadas - posición 81 a la 100- aportaron menos en las estrategias de RSC para mitigar los efectos del COVID-19.

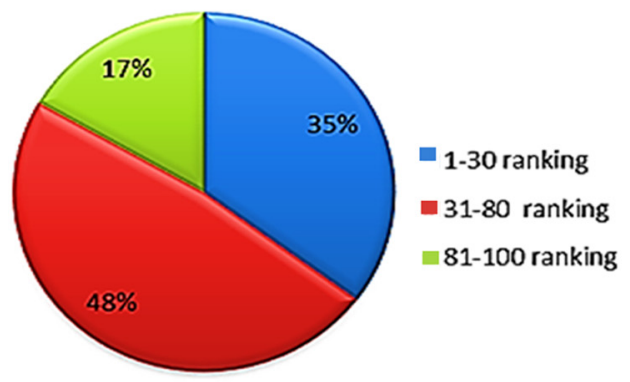

Gráfico 3. Mayor aporte social de empresas uruguayas por posición del ranking. Fuente: Elaboración propia.

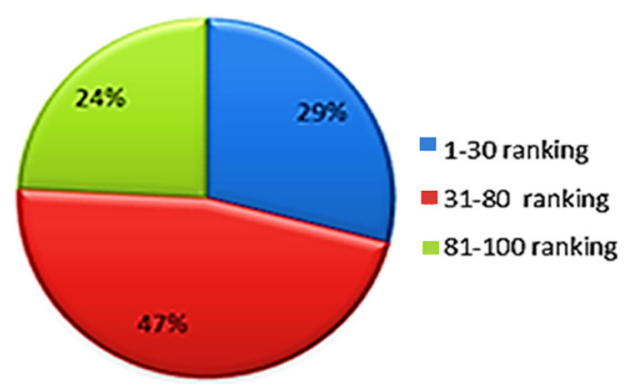

Gráfico 4. Mayor aporte social de empresas colombianas por posición del ranking. Fuente: Elaboración propia. 
En el caso de Uruguay, las empresas que revelan un aporte en mayor medida son de origen internacional mientras que en Colombia son nacionales, lo cual evidencia que, independientemente de su origen, todas las empresas pueden desarrollar prácticas RSC desde una perspectiva conductual, centrada en la capacidad adaptativa que deviene de las dinámicas del contexto. Es de destacar que en ambos países el sector industrial generó las mayores contribuciones para gestionar la crisis ocasionada por el COVID-19. Los gráficos 5 y 6 muestran en detalle las contribuciones por sectores económicos.

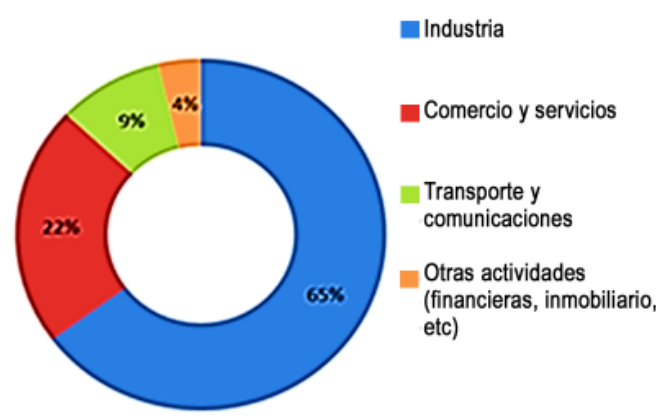

Gráfico 5. Mayor aporte social de empresas uruguayas por sector económico. Fuente: Elaboración propia.

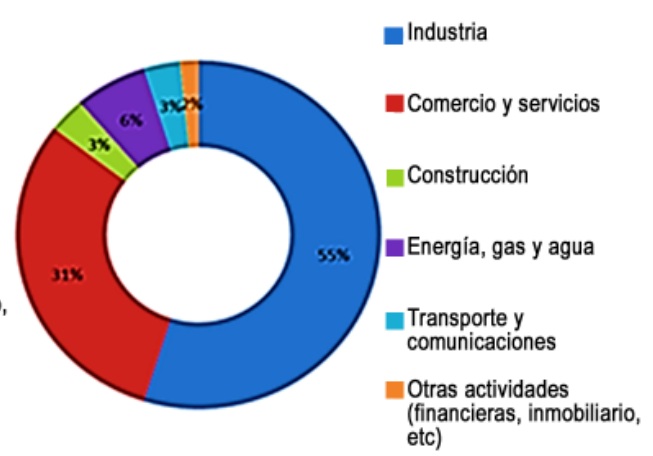

Gráfico 6. Mayor aporte social de empresas colombianas por sector económico. Fuente: Elaboración propia.

Complementario a lo representado en los anteriores gráficos, en la tabla 2 se sistematiza cómo las empresas más longevas, con más de 40 años de historia, y que, además, cuentan 1001 o más trabajadores en Uruguay y más de 5001 en Colombia, aportaron significativamente a la sociedad.

Junto a la tendencia en las empresas antiguas y, a su vez, más grandes de incorporar prácticas de gestión de la RSC entre sus objetivos y acciones, en cuanto al reporte, se aprecia que esta variable no es necesariamente determinante para la manifestación de la RSC, pero aun así, influye en dicho comportamiento. Las empresas más grandes y longevas representan el mayor número de reportantes y a su vez las que realizan contribuciones para superar las vulneraciones sociales, fenómenos ambientales y estrategias de desarrollo tecnológico.

Se pudo también comprobar que los reportes de sostenibilidad de las empresas estudiadas en su mayoría se caracterizan por emplear estándares e indicadores internacionales como los emitidos por el Global Reporting Initiative (GRI) y guías generadas por UNCTAD sobre contribuciones a los ODS, lo que 
Tabla 2. Relación antiguedad y tamaño con el aporte de las empresas.

\begin{tabular}{|c|c|c|c|}
\hline \multicolumn{2}{|c|}{ Uruguay } & \multicolumn{2}{|c|}{ Colombia } \\
\hline \multicolumn{4}{|c|}{ Antigüiedad } \\
\hline Hasta 10 años & 3 & Hasta 10 años & 1 \\
\hline De 11 a 40 años & 20 & De 11 a 40 años & 9 \\
\hline Más de 41 años & 29 & Más de 41 años & 52 \\
\hline Sin datos & 2 & & \\
\hline Total & 54 & Total & 62 \\
\hline \multicolumn{4}{|c|}{ Tamaño } \\
\hline $\begin{array}{c}\text { Hasta } 100 \\
\text { trabajadores }\end{array}$ & 4 & $\begin{array}{l}\text { Hasta } 2000 \\
\text { trabajadores }\end{array}$ & 8 \\
\hline $\begin{array}{l}\text { De } 101 \text { a1000 } \\
\text { trabajadores }\end{array}$ & 14 & $\begin{array}{l}\text { De } 2001 \text { a } 5000 \\
\text { trabajadores }\end{array}$ & 23 \\
\hline $\begin{array}{l}\text { Más de } 1001 \\
\text { trabajadores }\end{array}$ & 29 & $\begin{array}{l}\text { Más de } 5001 \\
\text { trabajadores }\end{array}$ & 31 \\
\hline Sin datos & 7 & & \\
\hline Total & 54 & Total & 62 \\
\hline
\end{tabular}

Fuente: Elaboración propia.

avala su calidad y pertenencia. Resulta interesante que las aportaciones de estas empresas durante la pandemia tendieron a estar relacionadas con la actividad económica que ejercen, como se relaciona a continuación:

- Las empresas productoras de combustible y alcohol realizaron donaciones de hipoclorito, jabón antibacterial, alcohol y alcohol en gel.

- Los ingenios azucareros en Colombia centraron sus donaciones en miles de litros de etanol para producir alcohol farmacéutico.

- Los fabricantes de productos de higiene personal y cuidado del hogar entregaron kits con estos artículos.

- Las empresas de transporte otorgaron viajes gratis a trabajadores de la salud. En Uruguay se destaca el uso del aeropuerto internacional de Carrasco, Uruguay, como un cine drive-in.

- El sector financiero y de seguros implementó estrategias asociadas a la reducción de tasas de interés para compras, en intereses de la cuota vigente de créditos; beneficios de cuenta de nómina para quienes quedaban desempleados, y ampliación de plazo de avances para las empresas del sector de alimentos y aquellas que realizaban donaciones a personas afectadas por la pandemia.

- Es de destacar que las empresas farmacéuticas en los países estudiados revelaban en sus informes y dentro de sus páginas WEB pactos de colaboración 
entre organizaciones del sector; realizaron pruebas diagnósticas y, en el marco de los acuerdos de la Federación Internacional de Farmacéuticas y Asociaciones (IFPMA) al unirse al Maratón Global de Compromiso de Respuesta al Coronavirus, dispusieron de herramientas y plataformas abiertas al público para que la comunidad científica las pudiera usar y, así mismo, de información fiable para que la sociedad conociera sobre la enfermedad y su contagio.

Dentro de la información divulgada en sus páginas WEB se encuentra que las empresas mayoritariamente en su rubro de informática incentivaron la creación de aplicaciones para mapear personas infectadas o relacionadas con enfermos de coronavirus.

Referente a la gestión del conocimiento se destaca que las consultoras empresariales dinamizaron la publicación de estudios referidos al impacto del COVID19. Se aúna a lo anterior guías y procedimientos para aplicación de la normativa legal que entró a regir con la pandemia y reportes periódicos sobre evaluación e impacto de estrategias implementadas por el COVID-19 en los negocios.

Se destaca dentro de las prácticas de la gestión de la RSC el voluntariado promovido por casi la totalidad de las empresas estudiadas, que incluía entregas de ayuda humanitaria y el compromiso solidario para mejorar la vida colectiva autorizando descuentos de días de salarios. Estas acciones evidencian que las contribuciones realizadas por la emergencia sanitaria consolidaron prácticas RSC en línea con los servicios en salud, sociales y económicos que reposaban dentro de las funciones del Estado (Crespo-Soler, et al., 2016), otorgándole a la RSC una nueva función frente a la noción de ciudadanía global.

\section{CONCLUSIONES Y DISCUSIONES}

En Uruguay, con una población de 3,5 millones de habitantes, en el momento en que se reportaron los primeros casos de COVID-19 el gobierno implementó medidas de contención; aun así, nunca se decretó un confinamiento obligatorio. En cambio en Colombia, con 50,88 millones de habitantes, al inicio de la pandemia el panorama fue totalmente distinto, toda vez que se inició decretando el estado de emergencia sanitaria cuando aparecieron los primeros casos, y dada la crisis política que incluía un estallido social, las medidas fueron poco eficaces; entonces la velocidad del contagio fue exponencial, lo cual se ha manifestado en un promedio de 129.000 muertes y más de 5,121 millones de personas que han sufrido el contagio.

La cultura ciudadana fue decisiva. Para el caso de Uruguay las personas acataron las medidas implementadas por el gobierno; según datos oficiales el $90 \%$ 
de los ciudadanos se quedaron en casa. En contraposición, en Colombia el estallido social y la crisis política entre gobierno nacional y gobernantes locales condujeron a que los ciudadanos tomaran a la ligera las implicaciones del COVID-19. Lo común en los dos países es el costo social y económico que representó el confinamiento, la reducción de las actividades de consumo, el congelamiento de puestos de trabajo y el incremento de la pobreza y la desigualdad.

Frente a este panorama social y económico, que para el caso colombiano incluye también el ámbito político, las 200 empresas radicas en Uruguay y Colombia con mejor índice de reputación según el Ranking Merco asumieron un rol estratégico en la generación de respuestas para mitigar algunas necesidades de las comunidades de sus áreas de influencia. Esta capacidad de reacción fue posible gracias a sus programas de RSC consolidados; por tanto, las más longevas generaron mayor aportación en la mitigación de las problemáticas asociadas con la pandemia, lo que permite ratificar la hipótesis H3.

El COVID-19 representó para las empresas, según los datos estudiados, nuevas formas de comprar, vender y consumir y el rol social que deben asumir, destacando la construcción de bienestar, la necesidad de implementar diversas estrategias de desarrollo sostenible, la inteligencia artificial como soporte para la toma de decisiones en los ámbitos ambientales y, desde luego, las inherentes a la estabilidad de sus colaboradores con programas de inclusión, el trabajo en casa, los procesos remotos mediados por tecnología e, incluso, la gestión temprana de las llamadas enfermedades silenciosas que trajo la pandemia en el ámbito psicosocial y ergonómico, y las asociadas a los estilos de vida. Lo anterior permite ratificar la hipótesis $\mathrm{H} 2$.

La contrastación de las variables evidencia que las organizaciones que asumieron la RSC como un facilitador para la generación de valor, la digitalización de las operaciones y la creación de nuevos modelos de negocio fueron aquellas que respondieron de mejor manera ante la pandemia, aceptando, por consiguiente, la hipótesis H1.

Como parte de la continuidad de esta investigación, y teniendo en cuenta los efectos actuales y los posteriores que se puedan generar por el COVID-19 a nivel global, se hace necesario en futuros estudios ampliar los métodos, las herramientas estadísticas y el número de empresas a estudiar para lograr un mayor análisis de los fenómenos abordados. Las capacidades de gestión desarrolladas por las empresas estudiadas hacen dar cuenta de nueva morfología de la RSC, cuya lógica es conectar con otros para mediar en problemáticas sociales y ambientales, llevando a resignificar los procesos de producción, el poder, la cultura y las experiencias de servicio. 
La literatura requiere analizar este nuevo entramado de sentido de la RSC como un sistema emergente, dinámico, abierto y capaz de expandirse. Se encuentra un importante desarrollo de nuevos códigos asociados al rol de la RSC, base de conocimiento y contribución al progreso científico y social, de forma especial por la resignificación del concepto de lo privado y la relación entre el mercado y el Estado, que se generó a partir de las prácticas de la gestión RSC que se adoptaron durante la pandemia por el COVID-19.

\section{REFERENCIAS}

Agle, B. R., Donaldson, T., Freeman, R. E., Jensen, M. C., Mitchell, R. K., \& Wood, D. J. (2008). Dialogue: Toward superior stakeholder theory. Business Ethics Quarterly, 18(2), 153-190.

Archel, P., Husillos, J., Larrinaga, C., \& Spence, C. (2009). Social disclosure, legitimacy theory and the role of the state. Accounting, auditing \& accountability journal, 22(November):1284-1307.

BBC. (2020). Coronavirus en Uruguay: la singular y exitosa estrategia del país para contener la pandemia sin cuarentena obligatoria. Obtenido el 7 de julio del 2020 de: https://www.bbc.com/mundo/noticias-america-latina-52837193

Boatright, J. R. (1996). Business ethics and the theory of the firm. Am. Bus. LJ, 34, 217.

Butler, H. N. (1988). The contractual theory of the corporation. Geo. Mason UL Rev., 11, 99.

CEPAL, N. (2018). Agenda 2030 y los Objetivos de Desarrollo Sostenible: una oportunidad para América Latina y el Caribe.

Crespo, C., Curvelo-Hassán, J. O., \& Ripol, V. (2016). Nuevas perspectivas de la gobernanza privada: un análisis desde las prácticas de responsabilidad social y ambiental de las empresas en Colombia. Revista Republicana, (21).

Fischer, J. (2004). Social responsibility and ethics: clarifying the concepts. Journal of Business ethics, 52(4), 381-390.

France 24. (2020). Colombia y el Covid-19, radiografía de un país que enfrenta la peor cara de la pandemia. Obtenido el 13 de agosto del 2020 de: https:/ / www.france 24.com/es/20200728-colombia-pandemia-covid19-crisis-sanitaria-hospitales

He, H., \& Harris, L. (2020). The Impact of Covid-19 Pandemic on Corporate Social Responsibility and Marketing Philosophy. Journal of Business Research, 116, 176182. 
Johns Hopkins University \& Medicine. (2020). See the latest data in your region. Obtenido el 14 de diciembre del 2020 de: https:/ / coronavirus.jhu.edu/region

Jonas, H. (2014). Technology and responsibility: reflections on the new tasks of ethics. In Ethics and emerging technologies (pp. 37-47). Palgrave Macmillan, London.

Kirk, C. P., \& Rifkin, L. S. (2020). I'll Trade You Diamonds for Toilet Paper: Consumer Reacting, Coping and Adapting Behaviors in the COVID-19 Pandemic. Journal of Business Research, 117, 124-131.

Maritz, A. (2020). A multi-disciplinary business approach to COVID-19: la trobe business school perspectives. International Journal of Organizational Innovation, 13(1).

Popkova, E., DeLo, P., \& Sergi, B. S. (2021). Corporate social responsibility amid social distancing during the COVID-19 crisis: BRICS vs. OECD countries. Research in International Business and Finance, 55, 101315.

PwC Uruguay. (2020). Novedades laborales COVID-19. Obtenido el 20 de julio del 2020 de: https:/ / www.pwc.com.uy/es/covid-19/pdfs-covid-19/via-rapida-novedades-laborales-covid19-abril.pdf

Qiu, S. C., Jiang, J., Liu, X., Chen, M. H., \& Yuan, X. (2021). Can corporate social responsibility protect firm value during the COVID-19 pandemic? International Journal of Hospitality Management, 93, 102759.

Rendtorff, J. D. (2009). Responsibility, ethics and legitimacy of corporations. Society and Business Review.

Watts, R. L., \& Zimmerman, J. L. (1978). Towards a positive theory of the determination of accounting standards. Accounting review, 53(1), 112-134.

Wittgenstein, L. (2014). Lecture on ethics. Edoardo Zamuner (Editor), Ermelinda Valentina Di Lascio (Editor), D. K. Levy (Editor). Editorial,? Wiley-Blackwell. Primera edición. 
